\title{
What Causes the Attention of Bitcoin?
}

\begin{abstract}
Bitcoin has received enormous attention both by the media and investors alike. But why has Bitcoin received such attention? This paper answers this question by examining the relationship between investor attention and Bitcoin fundamentals and finds that realized volatility and volume are both significant drivers of next day attention of Bitcoin.
\end{abstract}

Keywords: Investor Attention, Bitcoin, Google Search Volume Index, Realized Volatility

\section{Introduction}

In recent times, Bitcoin has been the subject of much attention, both by the media and investors. This surge in attention can be attributed to its innovative features, simplicity, transparency and its increasing popularity (Urquhart (2016)), but is also poses great challenges and opportunities for policy makers, economists, entrepreneurs, and consumers. The academic literature on Bitcoin is growing, with Cheah and Fry (2015) and Corbet et al. (2018) both documenting bubbles in the Bitcoin price, Urquhart (2016), Bariviera (2017) and Nadarajah and Chu (2017) all confirm the inefficiency of Bitcoin, Katsiampa (2017) showing that the best volatility model for Bitcoin is the AR-CGARCH model, Urquhart (2017) reports price clustering in Bitcoin, Phillip et al. (2018) shows that Bitcoin has many diverse stylized facts including long memory and heteroskedasticity while Baur et al. (2018) show that Bitcoin is a speculative investment and not an alternative currency or medium of exchange.

But a question yet to be answered in the literature is what factors have driven the attention of Bitcoin. To examine this, we employ Google Trends data as a proxy for investor attention to determine whether returns, realized volatility or volume are significant drivers of the attention of Bitcoin. We find that realized volatility and volume are significant drivers of next day 
investor attention but investor attention offers no significant predictive power in forecasting realized volatility, volume or returns.

\section{Data and Methodology}

We obtain attention data from Google Trends ${ }^{1}$ for the keyword "Bitcoin" and study the period 1st August 2010 to 31st July 2017. This sample period is chosen since before January 2010, the search volume is very low and many days consist of zero searches for "Bitcoin". Coincidentally, it also provides us with 7 full year of data which helps avoid any seasonality issues. The volume measure is based on the number of searches which were submitted within the USA. Since the data is relative in nature, we follow Dimpfl and Jank (2016) and standardize the search queries such that the average search frequency over the sample period equals one.

We use the search term "Bitcoin" to measure retail investors' attention to this index, since we find that this short name is the most widely used search term when investors are interested in bitcoin. Table 1 provides an overview of the search terms that have the highest correlation with the term "Bitcoin" according to Google Correlate. ${ }^{2}$ This table compares the level of search volume relative to the search term "Bitcoin" and we find that a number of different words have a very strong correlation with "Bitcoin". However, all of the highly correlated search terms have a lower search volume than "Bitcoin", and therefore do not add further information beyond our initial search. Seasonality is also an issue with search query data and therefore we follow Da et al. (2014) and regress the search query data on day-of-the-week and month-of-the-year dummies, however there is little evidence of significant seasonality pattern for trading days. ${ }^{3}$

We focus on the Bitstamp exchange as it is the most liquid and popular Bitcoin exchange in the US. Tick data is downloaded from

www. bitcoincharts.com where we aggregate the data to the 5-minute level to construct a time series of daily realized volatility $R V_{t}$ as introduced by

\footnotetext{
${ }^{1}$ Source: Google Trends (www.google.com/trends)

${ }^{2}$ Source: Google Correlate (www.google.com/trends/correlate/)

${ }^{3}$ As a robustness check, we filter our original series by taking the residual obtained from the regression on the day-of-the-week and month-of-the-year dummies. We then employ these filtered returns in our subsequent analysis and find qualitatively very similar results, which are available upon request from the corresponding author.
} 
Anderson et al. (2003) such that:

$$
R V_{t}=\sqrt{\sum_{j=1}^{n} r_{t, j}^{2}}
$$

where $r_{t, j}^{2}$ is the squared intraday log-price changes of the index and day $t$ during interval $j$ and $n$ is the number of such intraday return intervals. We compute these price changes over 5 minute intervals in order to circumvent the well documented microstructure effects (see Anderson et al. (2003)). To obtain daily volume and returns data, we aggregate the tick data to the daily level. ${ }^{4}$

Table 2 reports the descriptive statistics for the standardized search queries data, realized volatility, logarithmic trading volume as well as logarithmic returns. The standardized search queries has a maximum value of 3.67 and a minimum value of 0.07 indicating the large variation in the search query data. However, there is large positive skewness and excess kurtosis but when we take the logarithm of the search queries, we find that the excess skewness and kurtosis is less pronounced. The mean of the realized volatility is -6.36, with quite a large standard deviation of 1.24 as well as negative skewness. The mean logarithmic volume is 8.89 which indicates the liquidity of the Bitcoin market. Finally the returns show similar statistics as we would expect, namely positive mean return, negative skewness and a leptokurtic distribution.

In order to study the dynamics between search queries, realized volatility, trading volume and returns, we estimate vector autoregressive (VAR) models. Let $x_{t}$ be a vector that contains the variables of interest, then a $\operatorname{VAR}(k)$ reads as follows:

$$
x_{t}=c+\sum_{j=1}^{k} \beta_{j} x_{t-j}+\epsilon_{t}
$$

where $c$ is a vector of constants and $\epsilon_{t}$ is a vector of independent white noise innovations. The lag-length is determined using the Schwarz Bayesian information criterion. Model 1 investigates the dynamics between realized volatility and search queries $\left(x_{t}=\log S Q_{t} \log R V\right)$, while Model 2 sheds light

\footnotetext{
${ }^{4}$ We take logarithmic volume and returns in order to reduce skewness and kurtosis.
} 
on the interaction between search queries and trading volume $\left(x_{t}=\log S Q_{t}\right.$ $\log (\mathrm{ol})$ and Model 3 examines the relationship between search queries and returns $\left(x_{t}=\log S Q_{t} \log R e t\right)$.

\section{Empirical Results}

\subsection{Vector Autoregression and Impulse Response Results}

Table 3 displays the results of the three VAR models where the coefficient estimates are presented in Panel A, and the results of the Granger causality test are shown in Panel B. In Model 1, we find significant estimates of the autoregressive parameters for the realized volatility for lags 1, 4, 6 and 7 while search queries also show significant autoregressive terms for lags 1, 5 and 7 respectively. The estimation results also reveal that past search queries does not significantly influence realized volatility as the coefficient is only significant at the $10 \%$ level. This is also supported by the Granger causality test in Panel B which fails to reject the null hypothesis that search queries cause realized volatility. However we do find that past volatility significantly influences search queries at lag 1 indicating that an increase in volatility will lead to an increase in search queries the following day. Furthermore, the Granger causality test indicates that past volatility provides significant information about future search queries. Model 2 analysis the interaction of trading volume and search queries and similar to Model 1, the autoregressive search query term is significant at lag 1 . We also find that past volume provides significant information about future search queries. This suggests that when trading volume is high for Bitcoin, more information is sought from Google the following day. The Granger causality test also shows that trading volume Granger-causes search queries for Bitcoin. The interaction between returns and search queries is reported in Model 3 and interestingly shows that returns at lag 2 significantly provides information about search queries. This suggests that when returns of Bitcoin are high, the number of search queries increases but with a two day delay. There is also is no evidence of a significant autoregressive return term for Bitcoin. Also, Panel $\mathrm{B}$ shows that we can reject the null hypothesis that returns do not Granger cause search queries indicating that returns do Granger cause search queries.

To further examine what influences the attention of Bitcoin, Figure 1 presents the impulse response functions, where we employ the Cholesky decomposition. We can see that after a volatility, volume or returns shock, 
attention is elevated for a number of days, but is long lasting after a shock in returns.

\subsection{Subsample Results}

So far, our analysis has suggested that realized volatility, volume and returns all significantly influence future search queries. However this finding may not be stable over time and therefore we split our search queries data into two subsamples. To choose the split data, we employ the Bai and Perron (2003) test for and select the breakpoint with the greatest significance which corresponds to 28th October 2013. Therefore our first subsample covers 1st July 2012 to 27th October 2013 while the second subsample examines the period 28th October 2013 to 30th June 2017. The first subsample results are reported in Table 4 where we find that realized volatility has no significant influence on search queries. We also find that at lag 1, volume offers no significant influence on search queries although there is a negative significant effect at lag 2 indicating that lower volume one day influence search queries two days later. Also, returns have no significant influence on search queries at any lag, which indicates that our variables have no influence on future search queries. This is supported by the Granger causality tests which all fail to reject the null hypothesis in each case. Therefore from 1st July 2012 to 27th October 2013, realized volatility, volume and returns have no significant influence on future search queries. Table 5 presents the VAR results for the second subsample and shows that realized volatility significantly influences future search queries at lags 1, 2, 3 and 6 , while volume influences future search queries at lags 1,2 and 3 . We also find that returns at lag 2 significantly influence search queries indicating that there is a clear change in behaviour of our results over subsample. Since 28th October 2013, all three variables have been influencing search queries but not before. We also find, consistent with the full sample analysis, that search queries do not offer any significant predictive power in forecasting realized volatility, volume or returns.

\section{Conclusion}

This papers utilities Google Trends search queries to examine what drives the attention of Bitcoin. We find that previous day volatility and volume are significant drivers of attention of Bitcoin, as well as two days previous returns. However after splitting our data into two subsamples, we find that 
this is only the case from October 2013 and therefore our results indicate that investors are attracted to Bitcoin after large increases in volatility and trading volume of Bitcoin. 
Table 1: This table shows the top ten search terms that are most correlated with the search term 'bitcoin' during the sample period January 2010 to July 2017 on a weekly frequency. The second column provides the correlation coefficient from Google Correlate while the third column shows the search volume relative to the search volume for 'bitcoin'.

\begin{tabular}{cccc}
\hline Rank & Correlation & Relative Volume $(\%)$ & Search Term \\
\hline 1 & 0.9784 & $76.43 \%$ & bitcoin rate \\
2 & 0.9754 & $68.76 \%$ & current bitcoin \\
3 & 0.9746 & $83.89 \%$ & how bitcoin works \\
4 & 0.9740 & $77.81 \%$ & bitcoin usd \\
5 & 0.9736 & $71.63 \%$ & mining bitcoin \\
6 & 0.9732 & $78.20 \%$ & bitcoin trading \\
7 & 0.9694 & $93.71 \%$ & bitcoin currency \\
8 & 0.9693 & $81.73 \%$ & bitcoin? \\
9 & 0.9672 & $82.11 \%$ & bitcoin dollar \\
10 & 0.9666 & $60.32 \%$ & current bitcoin value \\
\hline
\end{tabular}

Table 2: This table reports the descriptive statistics of the standardized search queries, as well as the logarithmic search queries, logarithmic realized volatility, logarithmic volume and logarithmic returns.

\begin{tabular}{ccccccc}
\hline & Mean & Std.Dev & Max & Min & Skew & Kurtosis \\
\hline stan-SQ & 1.0000 & 0.5661 & 3.6724 & 0.0735 & 1.3353 & 3.7412 \\
log-SQ & -0.1796 & 0.6522 & 1.3009 & -2.6112 & -0.8797 & 0.8861 \\
log-RV & -6.3619 & 1.2372 & -0.0628 & -9.3531 & -0.1770 & 0.1973 \\
log-Volume & 8.8912 & 0.9267 & 11.8146 & 5.3762 & 0.7533 & 1.2581 \\
log-Returns & 0.0032 & 004916 & 0.3718 & -0.8219 & -3.0639 & 3.9501 \\
\hline
\end{tabular}


Table 3: This table displays the estimation results of three Vector Autoregressive Models for $\log$ realised volatility $(\log -R V)$, $\log$ search queries $(\log -S Q)$ and $\log$ trading volume $(\log -V O)$ for Bitcoin. Model 1 considers the dynamics between log-RV and $\log$-SQ and model 2 between $\log$-SQ and $\log$-VO, while Model 3 comprises of all variables. Panel A reports the coefficient estimates while Panel B provides the test statistics of the Granger causality test and $* * *, * *$ and $*$ indicate significance at the $1 \%, 5 \%$ and $10 \%$ levels respectively.

\begin{tabular}{|c|c|c|c|c|c|c|}
\hline \multicolumn{7}{|l|}{ Panel A: VAR Estimation } \\
\hline & \multicolumn{2}{|c|}{ Model 1} & \multicolumn{2}{|r|}{ Model 2} & \multicolumn{2}{|c|}{ Model 3} \\
\hline & $R V_{t}$ & $S Q_{t}$ & $S Q_{t}$ & $V O_{t}$ & $R_{t}$ & $S Q_{t}$ \\
\hline$S Q_{t-1}$ & $0.1625^{*}$ & $0.7352^{* * *}$ & $0.7411^{* * *}$ & 0.1152 & -0.0071 & $0.7899^{* * *}$ \\
\hline$S Q_{t-2}$ & $-0.1960^{*}$ & 0.0292 & 0.0319 & -0.1013 & 0.0058 & 0.0075 \\
\hline$S Q_{t-3}$ & -0.0065 & 0.0189 & 0.0264 & -0.0482 & -0.0072 & 0.0163 \\
\hline$S Q_{t-4}$ & -0.1388 & -0.0019 & 0.0103 & -0.0591 & $0.0181^{* *}$ & 0.0064 \\
\hline$S Q_{t-5}$ & $0.2456^{* *}$ & $0.0803^{* * *}$ & $0.0739^{* *}$ & 0.0871 & $0.0141^{* *}$ & $0.1443^{* * *}$ \\
\hline$S Q_{t-6}$ & 0.01676 & $0.0509^{*}$ & 0.0396 & 0.0583 & & \\
\hline$S Q_{t-7}$ & -0.0789 & $0.0576^{* *}$ & $0.0452^{*}$ & -0.0702 & & \\
\hline$R V_{t-1}$ & $0.5858^{* * *}$ & $0.0508^{* * *}$ & & & & \\
\hline$R V_{t-2}$ & 0.0271 & $-0.0303^{* * *}$ & & & & \\
\hline$R V_{t-3}$ & 0.0647 & -0.0106 & & & & \\
\hline$R V_{t-4}$ & $0.0650^{* *}$ & 0.0038 & & & & \\
\hline$R V_{t-5}$ & -0.0186 & -0.0022 & & & & \\
\hline$R V_{t-6}$ & $0.0570^{* *}$ & -0.0101 & & & & \\
\hline$R V_{t-7}$ & $0.0599^{* *}$ & -0.0085 & & & & \\
\hline$V O_{t-1}$ & & & $0.0686^{* * *}$ & $0.4651^{* * *}$ & & \\
\hline$V O_{t-2}$ & & & $-0.0392^{* * *}$ & 0.0240 & & \\
\hline$V O_{t-3}$ & & & -0.0139 & 0.0444 & & \\
\hline$V O_{t-4}$ & & & -0.0098 & -0.0591 & & \\
\hline$V O_{t-5}$ & & & -0.0048 & 0.0871 & & \\
\hline$V O_{t-6}$ & & & -0.0049 & $0.1114^{* * *}$ & & \\
\hline$V O_{t-7}$ & & & 0.0022 & $0.2134^{* * *}$ & & \\
\hline$R_{t-1}$ & & & & & 0.0350 & -0.0946 \\
\hline$R_{t-2}$ & & & & & $-0.1074^{* * *}$ & $0.3086^{* * *}$ \\
\hline$R_{t-3}$ & & & & & -0.0335 & $0.1806^{*}$ \\
\hline$R_{t-4}$ & & & & & $0.0531^{* *}$ & 0.1460 \\
\hline$R_{t-5}$ & & & & & $0.1051^{* * *}$ & 0.1070 \\
\hline Constant & $-0.8333^{* * *}$ & $-0.0581^{* * *}$ & -0.0058 & $0.7110^{* * *}$ & 0.0020 & $-0.0279^{* *}$ \\
\hline \multicolumn{7}{|l|}{ Panel B: Granger Causality Test } \\
\hline RV does not Granger Cause SQ & & $11.05^{* * *}$ & & SQ does not Granger Cause RV & & 1.56 \\
\hline Volume does not Granger Cause SQ & & $11.63^{* * *}$ & & SQ does not Granger Cause Volume & & 1.04 \\
\hline Returns does not Granger Cause SQ & & $3.62^{* * *}$ & & SQ does not Granger Cause Returns & & $2.60^{* *}$ \\
\hline
\end{tabular}


Table 4: This table displays the estimation results of three Vector Autoregressive Models for $\log$ realized volatility $(\log -R V)$, $\log$ search queries $(\log -S Q)$ and $\log$ trading volume $(\log -V O)$ for Bitcoin. Model 1 considers the dynamics between log-RV and $\log$-SQ and model 2 between $\log$-SQ and $\log$-VO, while Model 3 comprises of all variables. Panel A reports the coefficient estimates while Panel B provides the test statistics of the Granger causality test and ${ }^{* * *}, * *$ and $*$ indicate significance at the $1 \%, 5 \%$ and $10 \%$ levels respectively.

\begin{tabular}{|c|c|c|c|c|c|c|}
\hline \multicolumn{7}{|l|}{ Panel A: VAR Estimation } \\
\hline & \multicolumn{2}{|c|}{ Model 1} & \multicolumn{2}{|r|}{ Model 2} & \multicolumn{2}{|c|}{ Model 3} \\
\hline & $R V_{t}$ & $S Q_{t}$ & $S Q_{t}$ & $V O_{t}$ & $R_{t}$ & $S Q_{t}$ \\
\hline$S Q_{t-1}$ & $0.3522^{*}$ & 0.7931 & $0.7922^{* * *}$ & 0.2246 & -0.0142 & $0.7971^{* * *}$ \\
\hline$S Q_{t-2}$ & -0.3794 & 0.0522 & 0.0552 & $-0.2309^{* * *}$ & -0.0007 & 0.0520 \\
\hline$S Q_{t-3}$ & 0.0963 & -0.0326 & -0.0239 & 0.0635 & -0.0158 & -0.0300 \\
\hline$S Q_{t-4}$ & -0.2431 & -0.0114 & -0.0167 & 0.0294 & $0.0306^{*}$ & -0.0075 \\
\hline$S Q_{t-5}$ & 0.2952 & $0.1493^{* * *}$ & $0.1217^{* *}$ & 0.0772 & -0.0077 & $0.146^{* * *}$ \\
\hline$S Q_{t-6}$ & & & 0.0011 & -0.1230 & & \\
\hline$S Q_{t-7}$ & & & 0.0170 & 0.0213 & & \\
\hline$R V_{t-1}$ & $0.4969^{* * *}$ & 0.0160 & & & & \\
\hline$R V_{t-2}$ & 0.0287 & -0.0112 & & & & \\
\hline$R V_{t-3}$ & $0.0931^{*}$ & 0.0029 & & & & \\
\hline$R V_{t-4}$ & 0.0636 & -0.0029 & & & & \\
\hline$R V_{t-5}$ & 0.0412 & -0.0039 & & & & \\
\hline \multicolumn{7}{|l|}{$R V_{t-6}$} \\
\hline \multicolumn{7}{|l|}{$R V_{t-7}$} \\
\hline$V O_{t-1}$ & & & 0.0257 & $0.2625^{* * *}$ & & \\
\hline$V O_{t-2}$ & & & $-0.0296^{* *}$ & 0.0265 & & \\
\hline$V O_{t-3}$ & & & 0.00487 & -0.0374 & & \\
\hline$V O_{t-4}$ & & & -0.0146 & 0.0200 & & \\
\hline$V O_{t-5}$ & & & -0.0037 & -0.0307 & & \\
\hline$V O_{t-6}$ & & & -0.0028 & $0.1227^{* * *}$ & & \\
\hline$V O_{t-7}$ & & & 0.0175 & $0.2749^{* * *}$ & & \\
\hline$R_{t-1}$ & & & & & 0.0662 & 0.0179 \\
\hline$R_{t-2}$ & & & & & $-0.174 * * *$ & 0.2248 \\
\hline$R_{t-3}$ & & & & & -0.0620 & 0.2794 \\
\hline$R_{t-4}$ & & & & & 0.0254 & 0.1313 \\
\hline$R_{t-5}$ & & & & & $0.1614^{* * *}$ & 0.1168 \\
\hline Constant & $-1.5143^{* * *}$ & -0.0054 & & & 0.0059 & -0.0139 \\
\hline \multicolumn{7}{|l|}{ Panel B: Granger Causality Test } \\
\hline RV does not Granger Cause SQ & & 0.41 & & SQ does not Granger Cause RV & & 1.64 \\
\hline Volume does not Granger Cause SQ & & 1.23 & & SQ does not Granger Cause Volume & & 0.85 \\
\hline Returns does not Granger Cause SQ & & 1.06 & & SQ does not Granger Cause Returns & & 1.73 \\
\hline
\end{tabular}


Table 5: This table displays the estimation results of three Vector Autoregressive Models for $\log$ realized volatility $(\log -R V)$, $\log$ search queries $(\log -S Q)$ and $\log$ trading volume $(\log -V O)$ for Bitcoin. Model 1 considers the dynamics between log-RV and $\log$-SQ and model 2 between $\log$-SQ and $\log$-VO, while Model 3 comprises of all variables. Panel A reports the coefficient estimates while Panel B provides the test statistics of the Granger causality test and ${ }^{* * *},{ }^{* *}$ and $*$ indicate significance at the $1 \%, 5 \%$ and $10 \%$ levels respectively.

\begin{tabular}{|c|c|c|c|c|c|c|}
\hline \multicolumn{7}{|l|}{ Panel A: VAR Estimation } \\
\hline & \multicolumn{2}{|c|}{ Model 1} & \multicolumn{2}{|r|}{ Model 2} & \multicolumn{2}{|c|}{ Model 3} \\
\hline & $R V_{t}$ & $S Q_{t}$ & $S Q_{t}$ & $V O_{t}$ & $R_{t}$ & $S Q_{t}$ \\
\hline$S Q_{t-1}$ & -0.0110 & $0.6856^{* * *}$ & $0.6790^{* * *}$ & 0.0725 & 0.0005 & $0.7374^{* * *}$ \\
\hline$S Q_{t-2}$ & -0.0435 & 0.0423 & 0.0319 & -0.0008 & 0.0085 & -0.0191 \\
\hline$S Q_{t-3}$ & -0.1009 & -0.0258 & 0.0429 & $-0.173^{*}$ & -0.0003 & 0.0344 \\
\hline$S Q_{t-4}$ & -0.0777 & 0.0246 & 0.0410 & -0.097 & 0.0067 & 0.0168 \\
\hline$S Q_{t-5}$ & 0.1355 & 0.0676 & $0.0597^{*}$ & 0.1132 & -0.0096 & 0.0395 \\
\hline$S Q_{t-6}$ & 0.0784 & $0.1107^{* * *}$ & 0.0484 & $0.1959^{*}$ & -0.0065 & $0.0591^{*}$ \\
\hline$S Q_{t-7}$ & & & $0.0545^{* *}$ & -0.1253 & -0.0024 & $0.0862^{* * *}$ \\
\hline$R V_{t-1}$ & $0.6596^{* * *}$ & $0.0805^{* * *}$ & & & & \\
\hline$R V_{t-2}$ & 0.0227 & $-0.4391^{* * *}$ & & & & \\
\hline$R V_{t-3}$ & 0.0401 & $-0.0216^{* *}$ & & & & \\
\hline$R V_{t-4}$ & $0.0855^{* *}$ & 0.00976 & & & & \\
\hline$R V_{t-5}$ & -0.0320 & -0.1059 & & & & \\
\hline$R V_{t-6}$ & $0.0881 * * *$ & $-0.0220^{* * *}$ & & & & \\
\hline \multicolumn{7}{|l|}{$R V_{t-7}$} \\
\hline$V O_{t-1}$ & & & $0.0909^{* * *}$ & $0.5361^{* * *}$ & & \\
\hline$V O_{t-2}$ & & & $-0.0371^{* * *}$ & -0.0153 & & \\
\hline$V O_{t-3}$ & & & $-0.0211^{* *}$ & $0.0768^{* *}$ & & \\
\hline$V O_{t-4}$ & & & -0.0092 & $0.0783^{* *}$ & & \\
\hline$V O_{t-5}$ & & & -0.0132 & -0.0351 & & \\
\hline$V O_{t-6}$ & & & -0.0048 & $0.0857^{* * *}$ & & \\
\hline$V O_{t-7}$ & & & -0.0076 & $0.1550^{* * *}$ & & \\
\hline$R_{t-1}$ & & & & & -0.0150 & -0.1324 \\
\hline$R_{t-2}$ & & & & & $-0.0585^{* *}$ & $0.3811^{* * *}$ \\
\hline$R_{t-3}$ & & & & & -0.0015 & 0.1379 \\
\hline$R_{t-4}$ & & & & & $0.0759^{* * *}$ & $0.2129^{*}$ \\
\hline$R_{t-5}$ & & & & & $0.0479^{*}$ & 0.1700 \\
\hline$R_{t-6}$ & & & & & $0.0789^{* * *}$ & 0.0758 \\
\hline$R_{t-7}$ & & & & & 0.0389 & $0.1967^{*}$ \\
\hline Constant & -0.0054 & $-0.7893^{* * *}$ & 0.0297 & $1.129^{* * *}$ & -0.0004 & -0.0009 \\
\hline \multicolumn{7}{|l|}{ Panel B: Granger Causality Test } \\
\hline RV does not Granger Cause SQ & & $23.63^{* *}$ & & SQ does not Granger Cause RV & & 0.90 \\
\hline Volume does not Granger Cause SQ & & $15.58^{* * *}$ & & SQ does not Granger Cause Volume & & $1.88^{*}$ \\
\hline Returns does not Granger Cause SQ & & $3.05^{* * *}$ & & SQ does not Granger Cause Returns & & 1.49 \\
\hline
\end{tabular}



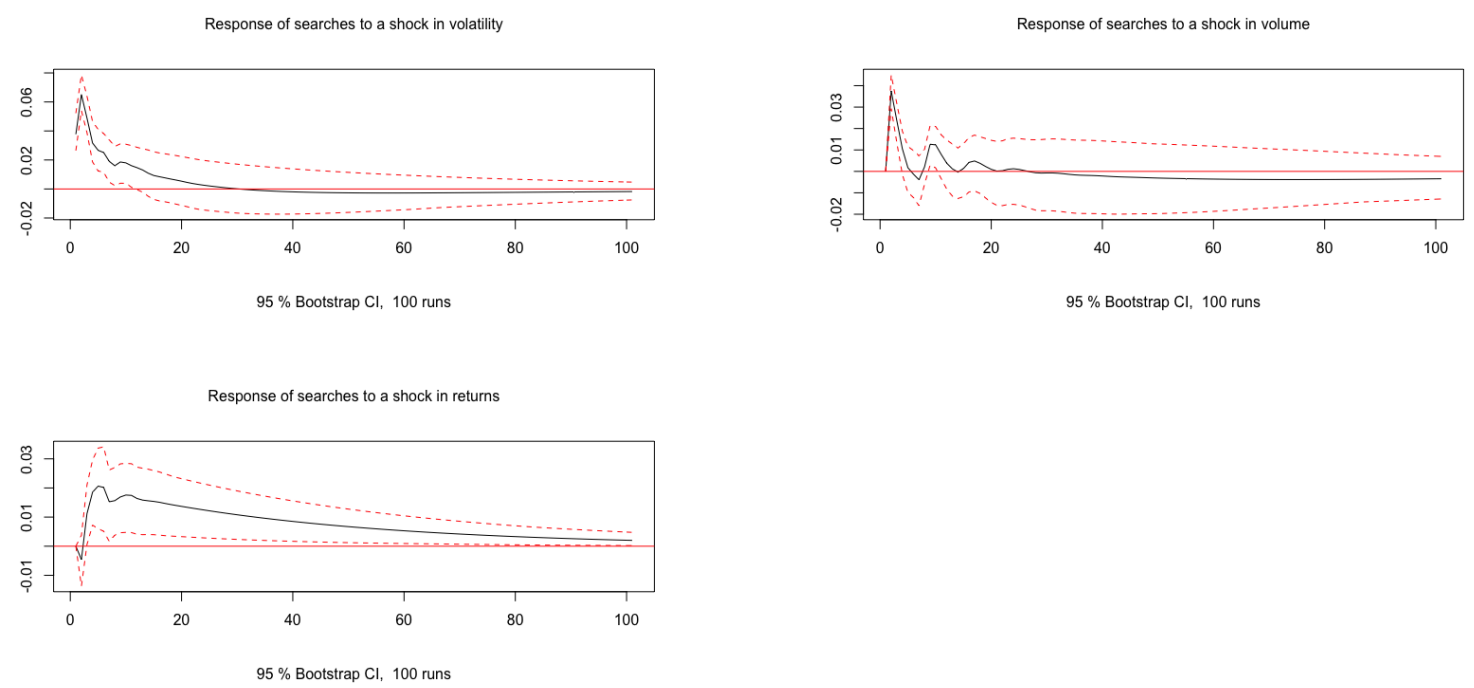

Figure 1: Impulse Response Functions for searches after a shock in volatility, volume and returns. 


\section{Bibliography}

Anderson, T. G., T. Bollerslev, F. X. Diebold, and P. Laybs (2003). Modeling and forecasting realized volatility. Econometrica 71, 579-625.

Bai, J. and P. Perron (2003). Computation and analysis of multiple structural change models. Journal of Applied Econometrics 18, 1-22.

Bariviera, A. F. (2017). The inefficiency of bitcoin revisited: A dynamic approach. Economics Letters 161, 1-4.

Baur, D. ., K. Hong, and A. D. Lee (2018). Bitcoin: Medium of exchange or speculative assets? Journal International Financial Markets, Institutions and Money, forthcoming.

Cheah, E. T. and J. Fry (2015). Speculative bubbles in bitcoin markets? An empirical investigation into the fundamental value of bitcoin. Economics Letters 130, 32-36.

Corbet, S., B. Lucey, and L. Yarovaya (2018). Datestamping the bitcoin and ethereum bubbles. Finance Research Letters, forthcoming.

Da, Z., J. Engelberg, and P. Gao (2014). The sum of all fears investor sentiment and asset prices. Review of Financial Studies 28, 1-32.

Dimpfl, T. and S. Jank (2016). Can internet search queries help to predict stock market volatility? European Financial Management 22, 171-192.

Katsiampa, P. (2017). Volatility estimation for bitcoin: A comparison of garch models. Economics Letters 158, 3-6.

Nadarajah, S. and J. Chu (2017). On the inefficiency of bitcoin. Economics Letters 150, 6-9.

Phillip, A., J. Chan, and S. Peiris (2018). A new look at cryptocurrencies. Economics Letters 163, 6-9.

Urquhart, A. (2016). The inefficiency of bitcoin. Economics Letters 148, $80-82$.

Urquhart, A. (2017). Price clustering in bitcoin. Economics Letters 159, $145-148$. 